\title{
A voltammetric electronic tongue for the quantitative analysis of quality parameters in wastewater
}

M. Carmen Martinez-Bisbal, ${ }^{*[a, b]}$, Edwin Loeff, ${ }^{[a]}$ Estela Olivas, ${ }^{[c]}$ Noèlia Carbó, ${ }^{[a]}$ F. Javier García Castillo, ${ }^{[c]}$ Javier López Carrero, ${ }^{[a]}$ Isabel Tormos, ${ }^{[a]}$ Francisco José Tejadillos, ${ }^{[c]}$ José Guillermo Berlanga ${ }^{\left[{ }^{[c]}\right.}$ Ramón MartínezMáñez ${ }^{[a, b, c]}$ Miguel Alcañiz, ${ }^{[a, e]}$ Juan Soto ${ }^{[a]}$

a Instituto Interuniversitario de Investigación de Reconocimiento Molecular y Desarrollo Tecnológico (IDM), Unidad Mixta Universitat Politècnica de València-Universitat de València, Valencia, Spain

b CIBER de Bioingeniería, Biomateriales y Nanomedicina (CIBER-BBN)

c Sociedad de Fomento Agrícola Castellonense, (FACSA), Castellón, Spain

d Departamento de Química, Universitat Politècnica de València, Valencia, Spain

e Departamento de Ingeniería Electrónica, Escuela Técnica Superior de Ingeniería del Diseño, Universitat Politècnica de València, Valencia, Spain

* e-mail: mamarbis@upvnet.upv.es

Received: ((will be filled in by the editorial sttaff))

Accepted: ((will be filled in by the editorial sttaff))

\begin{abstract}
The use of a voltammetric electronic tongue for the quantitative analysis of quality parameters in influent wastewater from a wastewater treatment plant (WWTP) that treats domestic and industrial wastewater is proposed. The electronic voltammetric tongue consists of a set of four noble electrodes (iridium, rhodium, platinum and gold) housed inside a stainless steel cylinder. These noble metals have high durability and are low maintenance-demanding, as required for developing future automated equipment. A pulse voltammetry study was conducted in 35 wastewater samples to determine ammonia $\left(\mathrm{NH}_{4}{ }^{+} \mathrm{N}\right)$, nitrates $\left(\mathrm{NO}_{3}{ }^{-} \mathrm{N}\right)$, total phosphate (tot-P), soluble chemical oxygen demand (CODs) and conductivity. These parameters were also determined in these samples by routine analytical methods in the WWTP laboratory. A partial least squares (PLS) analysis was run to obtain a model to predict each parameter. Twenty-five samples were included in the calibration set and 10 in the validation set. Calibration and validation sets were selected randomly, except for the extreme values of each parameter, which were included in the calibration set. Variable selection was performed on the voltammetric data using Genetic Algorithms in the calibration data set for each parameter. The electronic tongue showed good predictive power to determine the concentrations of $\mathrm{NH}_{4}{ }^{+}-\mathrm{N}, \mathrm{NO}_{3}{ }^{-}-\mathrm{N}$ and tot-P and CODs.
\end{abstract}

Keywords: Wastewater, Voltammetric electronic tongue, Water quality control, Variable selection, Genetic algorithms

DOI: 10.1002/elan.((will be filled in by the editorial sttaff))

\section{Introduction}

Environmental protection has become a growing social concern. Consequently, stringent quality requirements for effluent water have been established for wastewater treatment plants (WWTP) to reduce the impact on aquatic ecosystems [1]. In order to improve the efficiency of treatment processes so they meet these requirements, it is necessary to know the quality of influent wastewater. Wastewater quality is usually defined by the levels of diverse parameters related with main contaminants, such as chemical oxygen demand (COD), biological oxygen demand (BOD), ammonium, orthophosphate, nitrate, conductivity and $\mathrm{pH}$, among others. These parameters are usually determined by traditional laboratory analytical techniques. These analytical procedures are based mainly on sample collection and retrospective analyses, which makes their application to real-time monitoring and process controls difficult [2]. Off-line monitoring is suitable for monitoring slight changes over long time periods, when the time needed between the sample analysis and the reported results becomes less important [3]. On the contrary for real-time monitoring applications in a WWTP, on-line information about current influent water quality is required. In this scenario, interest in applying techniques to optimise and control WWTPs is increasing. For these purposes, automated systems and fast measurement procedures are highly valued. The use of commercially available sensors to monitor and control the biological processes that take place in WWTPs enhances pollutant removal efficiency [4]. Nevertheless, the strong investment and high maintenance costs of these specific sensors for each quality parameter are 
sometimes prohibitive for a medium- or small-sized WWTP.

Electronic tongues have emerged as a useful tool for qualitative and quantitative sample analyses. According to the agreed IUPAC definition, an electronic tongue is a "multisensory system, which consists of a number of low-selective sensors and uses advanced mathematical procedures for signal processing based on pattern recognition and/or multivariate data analysis" [5]. Basically, electronic tongues consist of an informationcollecting unit to be used in the aqueous phase, connected to a routine for multivariate data processing $[5,6]$. They use semi-specific sensors that produce a signal pattern which can be related to either a specific compound or a quality aspect of the sample [7,8]. Despite of the use of low-specificity sensors, their combination provides a huge information potential [6] for electronic tongues. A vast number of electronic tongues have been described based on different measurement techniques, but the majority are based on electrochemical measurements [6]. Among the diverse electrochemical techniques that can be implemented in an electronic tongue, the most versatile and robust is perhaps voltammetry because it is usually less influenced by electrical disturbances and has a favourable signal to noise ratio [9]. In voltammetric electronic tongues, a potential is applied to a working electrode. Then the redox active species are reduced or oxidised on the electrode surface and the resulting current, together with the current due to reorganisation charges on Helmholtz layers, are measured [6]. Given the simplicity and versatility of electronic tongues, and the possibility of them being implemented into automated online equipment, research efforts have been made to develop and use them in a wide range of applications, as in the food industry [10-14] and in environmental analyses $[15,16]$. In the water quality monitoring context, electronic tongues are appealing as they are easy to implement online and are low-cost [15]. However, very few studies have reported the potential use of electronic tongues in wastewater monitoring $[15,17]$.

Following our interest in using electronic tongues, we report herein a study on the ability of a simple voltammetric electronic tongue, based on four noble metals -iridium (Ir), rhodium ( $\mathrm{Rh})$, platinum $(\mathrm{Pt})$ and gold $(\mathrm{Au})$ - to determine several important parameters for wastewater quality control in WWTPs. The novelty of this manuscript compared to previous studies, is the more realistic approach based in several factors, to improve the development of a future system for the on-line monitoring. Regarding to the sensing system, this approach aims to acquire simplification in the sensor system using only noble metals. These noble metals, owing to their lower reactivity, have high durability and are less maintenance-demanding, as required for developing future automated equipment, compared to the non-noble metals. Moreover, the use of only four electrodes compared to previous published works with eight electrodes allows for a reduction in the number of variables to be considered, which saves data storage and computational time. In relation with the waste water origin, previous studies on the application of electronic tongues for wastewater monitoring have been carried out on influent wastewater samples from a WWTP pilot plant, equipped with a submerged anaerobic membrane bioreactor, and fed only with domestic wastewater [17]. Despite the advantages of these bioreactors, their efficient use is limited to domestic water treatment, with low pollutant contents, and located in places with a climate marked by mild temperatures. In this work, and in order to acquire a more generalised application, we studied influent wastewater samples from a WWTP equipped with an aerobic reactor, which treats domestic and industrial wastewater with high levels of pollutants. Moreover, the number of real samples has been increased to 35 and the samples have been studied native, without any dilution. Owing the complexity of analysis of these more polluted samples, variable reduction and variable selection using Genetic Algorithms have been used to select the most informative variables to improve the predictions. For all these reasons, this work aims for a more realistic context to show the application of electronic tongue for wastewater monitoring. With this experimental setting, we found that the voltammetric electronic tongue was able to predict levels of CODs, ammonia $\left(\mathrm{NH}_{4}{ }^{+}-\mathrm{N}\right)$, nitrates $\left(\mathrm{NO}_{3}{ }^{-} \mathrm{N}\right)$, total Phosphate (tot-P) and conductivity with acceptable accuracy and precision.

\section{Experimental}

\subsection{Wastewater sampling and analytical methods}

In order to study the influent wastewater composition, 35 samples were collected in a WWTP in the town of Almazora (Castellón,-E Spain) after the screening and degritter stages to eliminate solids and grease. The WWTP is equipped with an aerobic reactor, is feed with a mixture of domestic and industrial wastewater, and whose average volume is $6,400 \mathrm{~m}^{3} /$ day (year 2014). Wastewater samples were collected in a 15-day interval at different hours and on working days, and covered diverse wastewater compositions and, hence, different levels of domestic and industrial contaminants. The ranges of concentrations of the different parameters for the samples included in this study are shown in Table 1. Owing to changes in composition throughout the day and 
Table 1. The range of concentrations in wastewaters (units in ppm for the concentrations of $\mathrm{NH}_{4}{ }^{+}-\mathrm{N}, \mathrm{NO}_{3}{ }^{-}-\mathrm{N}$, tot-P and $\mathrm{COD}$ 's, and in $\mu \mathrm{S} / \mathrm{cm}^{2}$ for conductivity) for the set of 35 study samples is shown. The number of variables selected as the best set of variables by GA is also shown in this table for each parameter (GAVS). The adjusting parameters ( $\mathrm{R}^{2}, \mathrm{p} 1, \mathrm{p} 2$ and RMSEP) and the number of latent variables (LVs) are shown from the PLS prediction models in the validation set.

\begin{tabular}{lcccccccc}
\hline & Range & GAVS & LVs & $\mathrm{R}^{2}$ & $\mathrm{p} 1$ & $\mathrm{p} 2$ & RMSEP & RMSEP*100/max \\
\hline $\mathrm{NH}_{4}{ }^{+}-\mathrm{N}$ & $18.80-62.90$ & 28 & 9 & 0.815 & 0.832 & 6.08 & 5.91 & 9.39 \\
$\mathrm{NO}_{3}{ }^{-}-\mathrm{N}$ & $0.452-1.370$ & 41 & 6 & 0.701 & 0.978 & -0.05 & 0.135 & 9.83 \\
tot-P & $3.41-9.19$ & 34 & 10 & 0.686 & 0.900 & 1.21 & 1.06 & 11.58 \\
CODs & $148-1949$ & 25 & 9 & 0.664 & 0.946 & 72.75 & 176 & 14.74 \\
Cond & $1140-2340$ & 43 & 11 & 0.872 & 0.923 & 110 & 105 & 4.47
\end{tabular}

the diverse features of samples, the ranges of contaminants was quite wide (see Table 1). Samples were stored cold at $4^{\circ} \mathrm{C}$ and were then analysed with the electronic tongue in less than $48 \mathrm{~h}$.

Parallel routine analytical standardised tests (Lange cuvette test-system) were carried out to determine the contents of ammonia $\left(\mathrm{NH}_{4}{ }^{+}-\mathrm{N}\right)$, nitrates $\left(\mathrm{NO}_{3}{ }^{-} \mathrm{N}\right)$, total phosphate (tot-P) and chemical oxygen demand (CODs). Conductivity was determined with a conductimeter.

\subsection{Voltammetry measurements}

The electronic tongue system used in this study is based on pulse voltammetry, and was developed by the Instituto Interuniversitario de Investigación de Reconocimiento Molecular y Desarrollo Tecnológico (IDM), at the Universitat Politècnica de València (UPV, Spain). The electronic tongue is composed of electronic equipment, a software application that runs on a personal computer (PC) and a set of metallic electrodes. The electronic equipment applies voltage signals to electrodes. The temporal evolution of the current signal is collected for each working electrode and sent to the $\mathrm{PC}$ to be stored for further processing. A set of pulses is put together to form a pulse train in order to extract as much information as possible from the solution. [18] The details of this electronic equipment have already been published [19]. Following the methodology proposed by Winquist et al., [7] the electronic tongue device used in this work consisted in an array of four metallic working electrodes,
Ir, $\mathrm{Rh}, \mathrm{Pt}$ and $\mathrm{Au}$, with purity of $99.9 \%$ and a $2-\mathrm{mm}$ diameter. It was housed inside a homemade stainless steel cylinder, which was used at the same time as both the body of the electronic tongue system and the counter electrode. A more detailed description of the electrodes used can be found in Campos et al. 2012 and 2014 $[15,17]$. Electrodes were conditioned before the measurements were taken by mechanical polishing and immersion in an acidic solution. Then electrodes were rinsed with distilled water before measurements were taken. A saturated calomel electrode was used as the reference electrode.

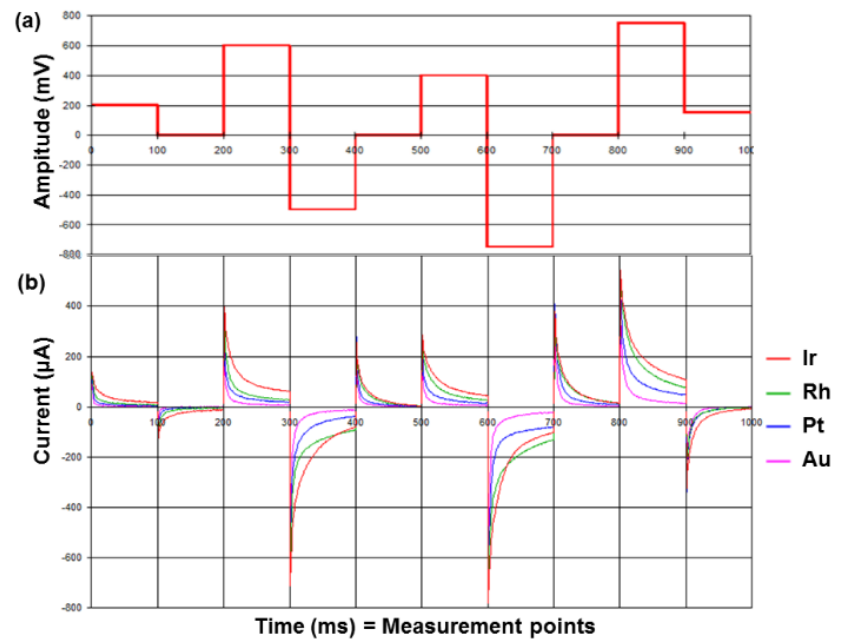

Figure 1: (a) Applied pulse sequence. The pulse sequence was the same for all the electrodes. (b) Response given by a wastewater sample included in the analysis. The data of all the electrodes are shown in the same figure. The abscissa axis shows the measured points and is equivalent to the time in $\mathrm{ms}$ as a pulse is described with 100 points and duration is $100 \mathrm{~ms}$. 
For each measurement, $50 \mathrm{~mL}$ of wastewater were introduced into a cell, with controlled temperature at $25.0 \pm 0.1^{\circ} \mathrm{C}$ (PolyScience). Voltammetric measurements were taken without adding any background electrolyte. The applied pulse sequence was the same for all four working electrodes and was composed of 10 pulses: 200 , $0,600,-500,0,400,-750,0,750$ and $150 \mathrm{mV}$. Each pulse was applied for $100 \mathrm{~ms}$. Current values collected per pulse and electrode were described by 100 measurements $(1 \mathrm{data} / \mathrm{ms})$. These pulses were designed according to the cyclic voltammetry information obtained in a previously published work [15]. In all, 4,000 points (10 pulses $\times 100$ points for pulse $\times 4$ electrodes) were recorded in each wastewater sample. Figure 1 shows the pulse sequence applied and the data collected in a sample.

\subsection{Data management and statistical analysis}

\subsubsection{Data sets for the study}

Thirty-five influent wastewater samples were used in this study. For each sample, three aliquots of $50 \mathrm{~mL}$ were measured to assess reproducibility. The data obtained in the three repetitions of each sample were averaged by considering the 4,000 points in each repetition. In order to evaluate the dispersion of each repetition, the average dispersion versus the median was calculated for each repetition $\mathrm{A}\left(\mathrm{ADM}_{\mathrm{A}}\right)$ on all 35 samples.

$$
A D M_{A}=\frac{\sum_{i=1}^{N}\left|M_{e i}-A_{i}\right|}{N}
$$

$\mathrm{ADM}_{\mathrm{A}}$ evaluates the difference between the measured value on each repetition $\left(\mathrm{A}_{\mathrm{i}}\right)$ and the median value $\left(\mathrm{M}_{\mathrm{ei}}\right)$ for each point/current value ( $\mathrm{N}$ points) of the signals. $\mathrm{ADM}_{\mathrm{A}}$ is used to rule out repetitions with high dispersion. Only the repetitions with a value of $\mathrm{ADM}_{\mathrm{A}}$ lower than $3 \mu \mathrm{A}$ were used to calculate the average. After the average, the data resulted in 4,000 data points per sample. Figure 2 shows a superposition of the data in the four electrodes for the 35 samples. The calibration (25 samples $\approx 70 \%$ ) and validation (10 samples $\approx 30 \%$ ) sets were selected randomly, except for the data with extreme values (maximum and minimum) of each parameter, which were included in the calibration set. The calibration and validation sets were the same for the statistical analysis in all the parameters.

\subsubsection{Data reduction and variable selection}

With the increasing ease of measuring multiple variables per object, the relevance of variable selection for data reduction and for improved interpretability is becoming more important [20]. Reducing obtained data sets also saves data storage and computational time [9]. The dimensionality of the data matrix considered herein was high $(4,000$ data points per sample). The dimensionality problem is typical in many fields of science [20] and it has been recognised that feature selection can be most beneficial to improve the model's predictive ability and to make it simpler [21]. Moreover, the model is easier to interpret and the studied system is better understood [20]. In the field of voltammetric tongues, several strategies have been used to reduce or select the number of variables. One approach is to select only a number of current values at certain fixed times to represent the different steps or pulses in pulse voltammetry experiments [18,22]. Data from voltammetric electronic tongues have also been simplified by modelling the response from electrodes by an equation that describes both the Faradic current and the charging current [23], or by an equation that describes the curve as a fourth-order polynomial approach for each pulse [19]. A chemical/physical model based on the voltammetric theory has been developed to extract interesting features of current transients. It revealed different information about species in solutions, which enhanced the separation ability in the PCA analysis done with different samples [9]. A discrete wavelet transform (DWT) and fast Fourier transform (FFT) have also been used to reduce the complexity of cyclic voltagrams from electronic tongues in wines samples $[12,24]$.

A different approach for variable reduction is to use genetic algorithms (GA), which is now used as a widespread subset search. GA are inspired by the biological evolution theory and by natural selection in the sense that the variables which yield fitted models showing high performance (or fitness), and were more likely to "survive" and to be included in variable sets in subsequent model refits. Furthermore, a mutation step ensures a certain level of randomness in the algorithm [20]. In the field of electrochemical measurements, GA

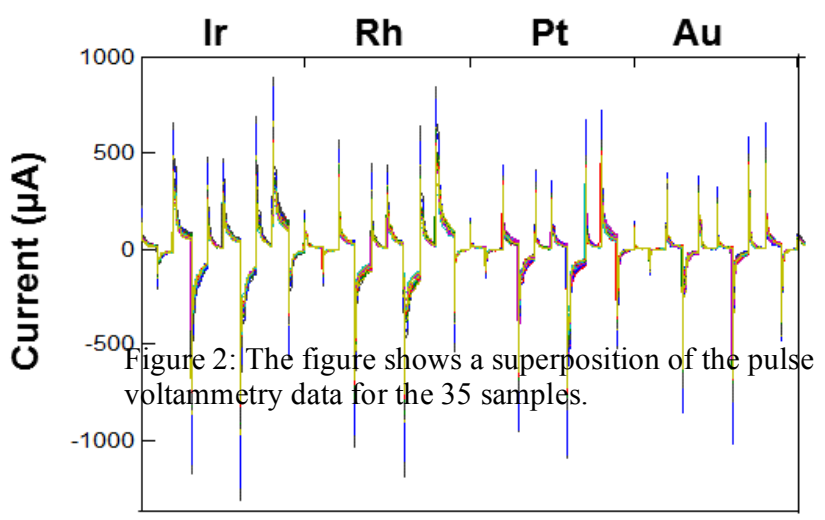


combined with PLS regression have been applied to reduce the considerable number of variables in polarography, stripping and square wave voltammetry [25] in order to determine different compounds and metals, and to characterise wine ageing [26]. In both cases the results obtained after variable selection were better than those obtained when considering the whole set of variables $[25,26]$.

In the present study, variable selection was performed using GA in the calibration set. Before applying GA to the original data set, it was considered that the performance of GA worsened when more than 200 input variables were used [27]. This is due to the fact that a high variables/object ratio increases the risk of overfitting, and the size of the search domain becomes too large $[26,27]$. In order to obtain an adequate data set size (4,000 current values for each sample), several data of interest were selected to represent the pulse by following the procedures of Winquist et al. 2011 and Ivarsson et al. 2005. In particular, of all the current values recorded in each pulse (100), two data sets were selected: 4 data per pulse (data recorded at 2, 5, 10 and $50 \mathrm{~ms} ; 4$ current values $\times 10$ pulses $\times 4$ electrodes $=160$ data per sample) and 5 data per pulse (data recorded at 2, 3, 5, 10 and $50 \mathrm{~ms} ; 5$ current values $\times 10$ pulses $\times 4$ electrodes $=$ 200 data per sample). This reduced the 4000 data in the original set to 160 data (a variable reduction of $96 \%$ ) or to 200 data (a variable reduction of 95\%) for each sample. After this variable reduction, GA variable selection was performed with both data sets (160 and 200 points) with the calibration set (25 samples) for each parameter $\left(\mathrm{NH}_{4}{ }^{+}-\mathrm{N}, \quad \mathrm{NO}_{3}{ }^{-}-\mathrm{N}\right.$, tot-P, $\mathrm{CODs}$ and conductivity) to further reduce the number of variables using the software PLS_Toolbox Solo 8.0 (Eingenvector Research, Inc.) for chemometrics analyses. Data were preprocessed by performing an autoscale (mean centring and scaling each variable to the unit standard deviation). Then GA were performed directly with the whole set of 160 data. However, the set of 200 data was split into two subsets with 100 variables each (the Ir and $\mathrm{Rh}$ data in one subset, and the $\mathrm{Pt}$ and $\mathrm{Au}$ data in the other subset) to reduce the variables/objects ratio, and to thus improve the ability of GA for variable selection. The results of the GA selected variables from the two subsets of 100 were combined as a final set of selected variables.

\subsubsection{PLS analysis}

A multivariate analysis was performed by the PLS [28] method and using the software PLS_Toolbox Solo 8.0 (Eigenvector Research, Inc.) for chemometrics analysis before the analysis, data were autoscaled. In order to evaluate the adequacy of the experimental data and to select the quantity of latent variables, a cross-validation was performed before building the model. Venetian Blinds was the method used in the cross-validation. The minimum value for the cross-validation error was used to select the number of latent variables for the model. Then the obtained model was applied to the set of validation samples to predict the values of all the parameters of interest: $\mathrm{NH}_{4}{ }^{+}-\mathrm{N}, \mathrm{NO}_{3}{ }^{-}-\mathrm{N}$, tot-P, CODs and conductivity. The models were evaluated to determine the overfit risk by running Permutation tests (Pairwise Wilcoxon, Signed Rank test and Rand $t$-test). Finally, model evaluation was made by comparing real versus predicted concentrations using the correlation coefficient $\left(\mathrm{R}^{2}\right)$, the root mean square error of prediction (RMSEP), and slope (p1) and intercept ( $\mathrm{p} 2$ ) for the validation set (from $\mathrm{y}=\mathrm{p} 1 \cdot \mathrm{x}+\mathrm{p} 2$ based on a simple lineal model).

Five data sets were evaluated for each parameter with PLS, and according to the different variable reduction and selection trials. The five data sets were: a) the whole set of data (4,000 current values); b) the data reduced to 5 points per pulse (200 current values); c) the data reduced to 4 points per pulse (160 current values); d) the data selected by GA from the set of 5 points per pulse; e) the data selected by GA from the set of 4 points per pulse. The final number of data considered in the GA differed for each parameter and depended on the best result achieved in GA performance.

\section{Results and Discussion}




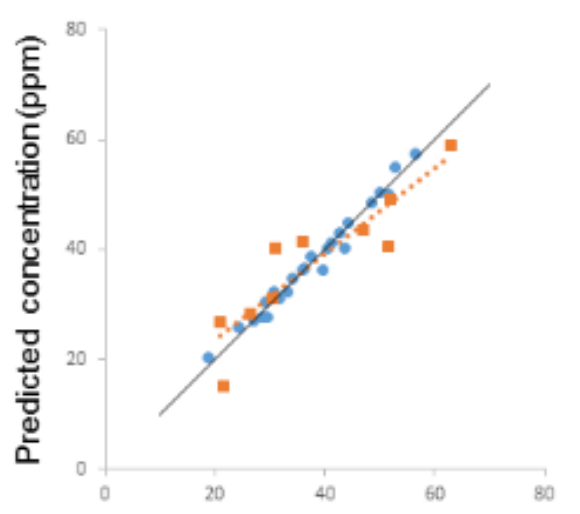

$\mathrm{NH}_{4}{ }^{+}=\mathrm{N}$ Measured concentration $(\mathrm{ppm})$

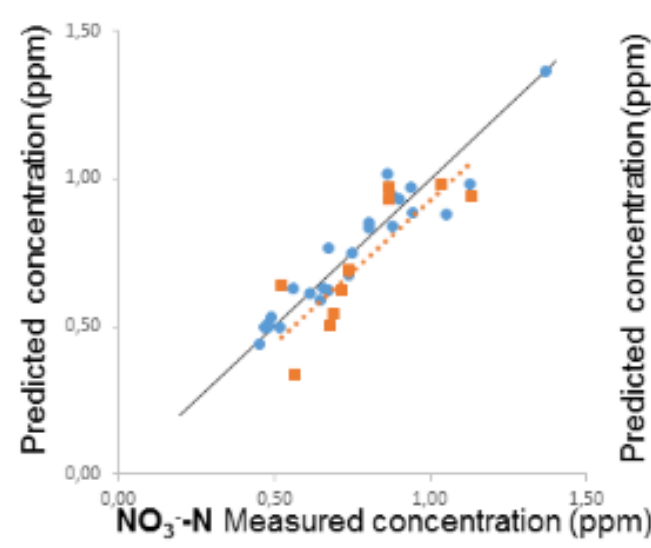

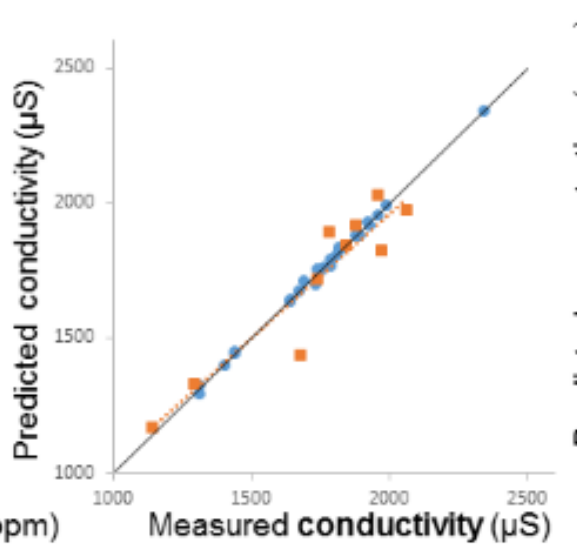

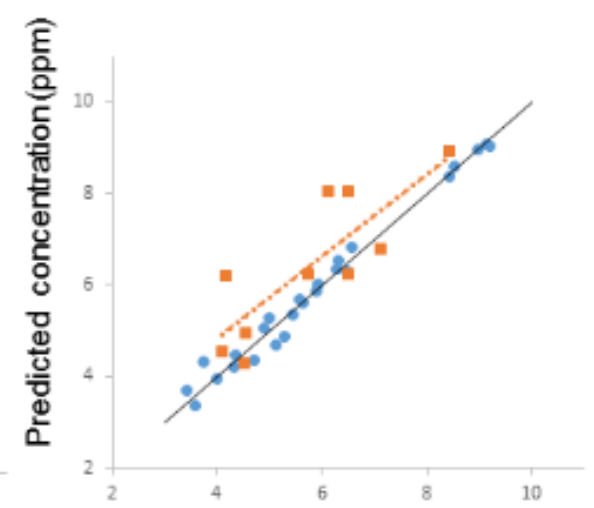

tot-P Measured concentration (ppm)

A PLS Figure 3: The PLS prediction results are provided in this figure for $\mathrm{NH}_{4}^{+}-\mathrm{N}$, conductivity, tot-P, $\mathrm{NO}_{3}{ }^{-}-\mathrm{N}$ and $\mathrm{DQO}$. Plots show the 25 samples of the calibration set as blue circles and the prediction for 10 samples in the validation set as red squares. The 1:1 line (solid bla line) and the fitting line for the validation set (dashed line in red) are also displayed.

analysis was used to obtain a correlation between voltammetric measurements using the electronic tongue and the contents of $\mathrm{NH}_{4}{ }^{+}-\mathrm{N}, \mathrm{NO}_{3}{ }^{-}-\mathrm{N}$, tot-P, COD, according to analytical tests and conductivity. The best results were obtained when using the data selected by GA. The values of $\mathrm{R}^{2}, \mathrm{p} 1, \mathrm{p} 2$ and RMSEP are shown in Table 1 for the set with best PLS performance in each parameter, together with the range of measurements, the number of GA selected variables and the RMSEP in relation to the maximum value within the range of each parameter. This last data provides an idea of the percentage of error in predictions. For all the parameters, the GA data set improved the PLS results for predictions. The GA ran from the initial set of 200 data obtained the best PLS performances for $\mathrm{NO}_{3}{ }^{-} \mathrm{N}$, tot-P and conductivity using 41, 34 and 43 variables, respectively (which meant a variable reduction of $98.30 \%, 99.15 \%$ and $98.93 \%$, respectively). The GA run from the initial set of 160 data led to the best PLS performances for $\mathrm{NH}_{4}{ }^{+}-\mathrm{N}$ and CODs using 28 and 25 variables, respectively (which meant a variable reduction of $99.30 \%$ and $99.37 \%$, respectively). The Permutation Test calculated in all the models showed that the models built from GA were unlikely over-fit models.

The PLS calibration set and prediction set for $\mathrm{NH}_{4}{ }^{+}-\mathrm{N}$, conductivity, $\mathrm{NO}_{3}{ }^{-}-\mathrm{N}$, tot-P and CODs are shown in Figure 3 (predicted parameter vs. measured parameter). Figures 3 offer an idea of the accuracy and precision in the prediction model applied to the validation set.

Numerically, an idea of the accuracy and precision of predictions can be obtained when linearly fitting the experimental points calculated in the predicted group according to the model in the calibration set. The slope (p1) and the intercept with the $y$ axis (p2) of the linear fitting of the predicted vs. real data in the validation set $(y=p 1 x+p 2)$ were related to accuracy in prediction. The model was much better as p1 approached 1. RMSEP 
deals with model precision and the model was much better as RMSEP approached 0 .

The data in Table 1 indicate that in all cases predictions had higher $\mathrm{p} 1$ values than 0.8 and 0.9 for parameters $\mathrm{NO}_{3}{ }^{-}-\mathrm{N}$, tot-P, COD's and conductivity. According to the RMSEP values and the range of measurements for each parameter, it was established that the parameters in the validation set could be determined with errors lower than $10 \%$. The level of accuracy and precision of these results was acceptable if we bear in mind that samples were quite complex and were analysed by simple equipment based on the use of an electronic tongue with metallic electrodes.

An important point to achieve this prediction performance was the selection of data of interest from the whole pulse, previous to GA variable selection. As it has been explained, it is recommended to perform GA with no more than 200 input variables, but the data sets here considered were originally of 4000 current values for each sample. In a first step to reduce the dimensionality, a reduced number of current values was selected from the whole pulse. The selection was done, as already mentioned, according to previously published data, and taking into account points to give information of both Faradic and non-Faradic contributions to the signal. This selection would enable to detect both electroactive and not electroactive species as phosphate compounds, which are also of interest in this case.

Finally, a more realistic approach for the quantitative analysis of quality parameters in wastewater by electronic tongue has been shown. This approach is based in diverse factors which are the study samples with a high level of pollutants, the use of a sensing system composed by only noble metals with high durability and a low maintenance demanding, the saving in data storage and computational time by the reduction in the number of variables preserving representative information of Faradic and nonFaradic contributions, and the use of GA for variable selection to improve the predictions. This electronic tongue is low-cost, offers high durability and cuts the analysis time from hours/days to minutes.

\section{Conclusions}

The use of a simple electronic tongue combined with variable reduction strategies for multivariante analysis is described herein to analyse wastewater quality parameters in highly polluted influent real wastewater samples from a WWTP equipped with an aerobic reactor which is fed with domestic and industrial wastewaters. The parameters analysed in this study $\left(\mathrm{NH}_{4}{ }^{+}-\mathrm{N}, \mathrm{NO}_{3}{ }^{-}-\mathrm{N}\right.$, tot-P, CODs and conductivity) are very important for planning wastewater treatment of either domestic or industrial origin. The equipment that we used consisted of an electronic voltammetric tongue with only four noble metal electrodes ( $\mathrm{Ir}, \mathrm{Rh}, \mathrm{Pt}$ and $\mathrm{Au}$ ) housed inside a stainless steel cylinder. Only noble metals were used since they have higher durability and are less maintenance-demanding compared to non-noble metals, and also with the aim to reduce the number of data to be analysed to save computational time and data storage. Data dimensionality lowered in more than $98 \%$ by selecting a set of representative data per pulse and performing GA variable selection on them. The PLS studies indicated that the information obtained using an electronic voltammetric tongue with only four noble metals could be used to make estimations of these parameters rapidly and with acceptable accuracy and precision. These results suggested that simple electronic tongues based only on noble metals can be applied in WWTPs to improve the efficiency of domestic and industrial wastewater treatment processes. Such equipment is inexpensive, needs very little maintenance, offers high durability and can be implemented in situ to offer continuous water quality monitoring.

This information on influent wastewater is very useful for optimising WWTP operations and for saving costs during treatment processes. Moreover, the use of lowmaintenance electrodes opens up a possibility for future automated developments.

\section{Acknowledgements}

The financial support for this work was provided by the Spanish Ministry of Economy and Competitivity though Project e-TONGUE4WAT (IPT-2012-0069-310000) INNPACTO and by European Union FEDER funds. Financial support from the Spanish Government (Project MAT2012-38429-C04) and the Generalitat Valencia (Project PROMETEOII/2014/047) is also gratefully acknowledged.

\section{References}

[1] G. Olsson, Water Res. 2012, 46, 1585-1624.

[2] A. Bonastre, R. Ors, J. V. Capella, M. J. Fabra, M. Peris, TrAC - Trends Anal. Chem. 2005, 24, 128-137. 
[3] A. Mimendia, J. M. Gutiérrez, L. Leija, P. R. Hernández, L. Favari, R. Muñoz, M. del Valle, Environ. Model. Softw. 2010, 25, 1023-1030.

[4] J. D. Jang, J. P. Barford, Lindawati, R. Renneberg, Biosens. Bioelectron. 2004, 19, 805-812.

[5] Y. Vlasov, a. Legin, a. Rudnitskaya, C. Di Natale, a. D’Amico, Pure Appl. Chem. 2005, 77, 1965-1983.

[6] F. Winquist, Microchim. Acta 2008, 163, 3-10.

[7] F. Winquist, P. Wide, I. Lundström, Anal. Chim. Acta 1997, 357, 21-31.

[8] Y. G. Vlasov, a. V. Legin, a. M. Rudnitskaya, a. D'Amico, C. Di Natale, Sensors Actuators, B Chem. 2000, 65, 235-236.

[9] S. Holmin, P. Spångeus, C. Krantz-Rülcker, F. Winquist, Sensors Actuators, B Chem. 2001, 76, 455-464.

[10] I. Campos, R. Masot, M. Alcañiz, L. Gil, J. Soto, J. L. Vivancos, E. García-Breijo, R. H. Labrador, J. M. Barat, R. Martínez-Mañez., Sensors Actuators, B Chem. 2010, 149, 71-78.

[11] P. Ivarsson, S. Holmin, N. E. Höjer, C. Krantz-Rülcker, F. Winquist, Sensors Actuators, B Chem. 2001, 76, 449-454.

[12] X. Cetó, J. M. Gutiérrez, L. Moreno-Barón, S. Alegret, M. Del Valle, Electroanalysis 2011, 23, 72-78.

[13] Z. Wei, J. Wang, X. Zhang, Electrochim. Acta 2013, 88, 231-239.

[14] Z. Wei, J. Wang, Anal. Chim. Acta 2011, 694, 46-56.

[15] I. Campos, M. Alcañiz, D. Aguado, R. Barat, J. Ferrer, L. Gil, M. Marrakchi, R. Martínez-Mañez, J. Soto, J.-L. Vivancos, Water Res. 2012, 46, 2605-2614.
[16] F. Winquist, J. Olsson, M. Eriksson, Anal. Chim. Acta 2011, 683, 192-197.

[17] I. Campos, A. Sangrador, R. Bataller, D. Aguado, R. Barat, J. Soto, R. Martínez-Máñez, Electroanalysis 2014, 26, 588-595.

[18] P. Ivarsson, M. Johansson, N.-E. Höjer, C. KrantzRülcker, F. Winquist, I. Lundström, Sensors Actuators B Chem. 2005, 108, 851-857.

[19] E. Garcia-Breijo, R. M. Peris, C. O. Pinatti, M. A. Fillol, J. I. Civera, R. B. Prats, IEEE Trans. Instrum. Meas. 2013, 62, 424-431.

[20] T. Mehmood, K. H. Liland, L. Snipen, S. Sæbø, Chemom. Intell. Lab. Syst. 2012, 118, 62-69.

[21] E. V. Thomas, Anal. Chem. 1994, 66, 795A-804A.

[22] F. Winquist, J. Olsson, M. Eriksson, Anal. Chim. Acta 2011, 683, 192-197.

[23] T. Artursson, P. Spångeus, M. Holmberg, Anal. Chim. Acta 2002, 452, 255-264.

[24] X. Cetó, A. González-Calabuig, J. Capdevila, A. PuigPujol, M. del Valle, Sensors Actuators B Chem. 2015, 207, 1053-1059.

[25] A. Herrero, M. Cruz Ortiz, Anal. Chim. Acta 1999, 378, 245-259.

[26] N. Prieto, P. Oliveri, R. Leardi, M. Gay, C. Apetrei, M. L. Rodriguez-Méndez, J. a. De Saja, Sensors Actuators, B Chem. 2013, 183, 52-57.

[27] R. Leardi, A. Lupiáñez González, Chemom. Intell. Lab. Syst. 1998, 41, 195-207.

[28] P. Geladi, B. R. Kowalsky, Anal. Chim. Acta 1986, 185, $1-17$. 